Research Article in Space

\title{
Making Mars Habitable to Humans
}

\author{
Maxwell Luo ${ }^{1, *}$ \\ ${ }^{1}$ Baltimor County Public Schools, Perry Hall, MD 21128, USA \\ *Correspondence: maxwellwluo@gmail.com
}

(Received: 08/18/2020; Accepted: 09/21/2020; Published: 10/09/2020)

DOI: https://doi.org/10.37906/isteamc.2020.7

\begin{abstract}
This article summarizes and discusses approaches to make Mars habitable to human beings. The human being needs key requirements to survive, which are food, oxygen, water, and shelter. The techniques of photocatalytic water splitting technique and electrolysis are compared for oxygen generation. The hydroponic and aquaponics, vertical farming, and aeroponics are analyzed in this article to viable solutions to providing food on Mars. The shelter is another mandatory requirement for humans to survive on Mars. Techniques and approaches such as 3D printing, inflatable shelters, caves, and lava tubes are discussed in the article. To fully colonize Mars, terraforming is recommended as it creates an Earth-like environment and allows humans to live on Mars as if they live on Earth. However, it could take hundreds of years to achieve the terraforming goal. The combined use of all these techniques can make a suitable environment for the life of humans on Mars, and the goal of colonizing Mars is feasible and achievable.
\end{abstract}

Keywords: mars, colonization, habitat, terraforming

\section{Introduction}

Mars is known as the "red planet" has aroused questions to human beings since the very beginning of human civilization history. From the time of Egyptians to the present, Mars has sparked curiosity and imagination. Mars is named after the Roman god of war. It is only half the size of Earth, only has 1/10 of Earth's mass, and has $38 \%$ of Earth's gravity. So, a 100-pound person would only weigh 38 pounds on Mars. Its volume is $15 \%$ of Earth's, and it is 142,000,000 miles away from the sun, while Earth is only 92,000,000 miles away. Mars has 2 moons, Phobos (fear) Deimos (terror), which are minor Greek gods. Mars is very cold, having $-81 \mathrm{~F}$ as its average temperature. Mars has an atmosphere that is thin compared to the Earth, but thick compared to the moon. Its atmosphere is mostly made of carbon dioxide, and a small amount of water vapor. While Earth's atmosphere is made of nitrogen, oxygen, argon, and others. An average Earth day is 23 hours and 56 minutes, while a Mars day is 24 hours and 37 minutes.

Why should we colonize Mars instead of the other planets in the solar system? Mars is the current best planet except for the Earth for us to survive on. Mars is the closest planet to Earth and is the only available terrestrial planet that is not burning hot. Mars has water, and a thicker atmosphere to protect from comets, meteors as well as cosmic radiation, and geological history once suggests that Mars had an environment like Earth's. Mars has the potential over other Terraforming targets like the moon or other planets. Now, we start to dream that we will colonize Mars in the near future. Some scientists say we will terraform Mars 
to make it more like our Earth. While others say we should just put shelter on Mars for our human beings and leave the rest of the "red planet" as is. Other disputes are over many subjects like how will we eat, how will our shelter be built, what will our shelter be built from, or should we even colonize Mars?

We should colonize Mars, and I will explain in detail in this article on how we will make Mars habitable to humans.

\section{Conditions on Mars}

Mars is a rocky place as shown in (Figure 1). NASA gives a good description of the red planet: "Its surface is rocky, with canyons, volcanoes, dry lake beds and craters all over it. Red dust covers most of its surface. Mars has clouds and wind just like Earth. Sometimes the wind blows the red dust into a dust storm. Tiny dust storms can look like tornadoes and large ones can be seen from Earth. Mars' large storms sometimes cover the whole planet" (Wild, 2017). These geographic features on Mars show that it is very cold and lifeless. Rocks on Mars are red in color due to iron oxide, the same substance that makes blood red. Many trenches and volcanoes are on the surface. Mars has ice caps on the poles, like Earth. Mars has an oxygen concentration of $0.1 \%$ in its atmosphere, while oxygen concentration is $21 \%$ in the atmosphere on Earth.

As we know, Mars has less gravity, which is only 38\% of Earth's gravity. Mars also has dust storms as mentioned by NASA, and they could be a potential problem that can cover solar panels and cut off energy sources. Mars has no magnetic field, meaning that compasses would not work. But more importantly, Mars cannot shield the radiation which is dangerous to life without a magnetic field. Mars has a very thin atmosphere and thus it does not have enough greenhouse effect to maintain the surface temperature. Mars cannot block large objects like big meteors. Therefore, it will need some major adjustments for it to be habitable, meaning it will be no easy task colonizing Mars.

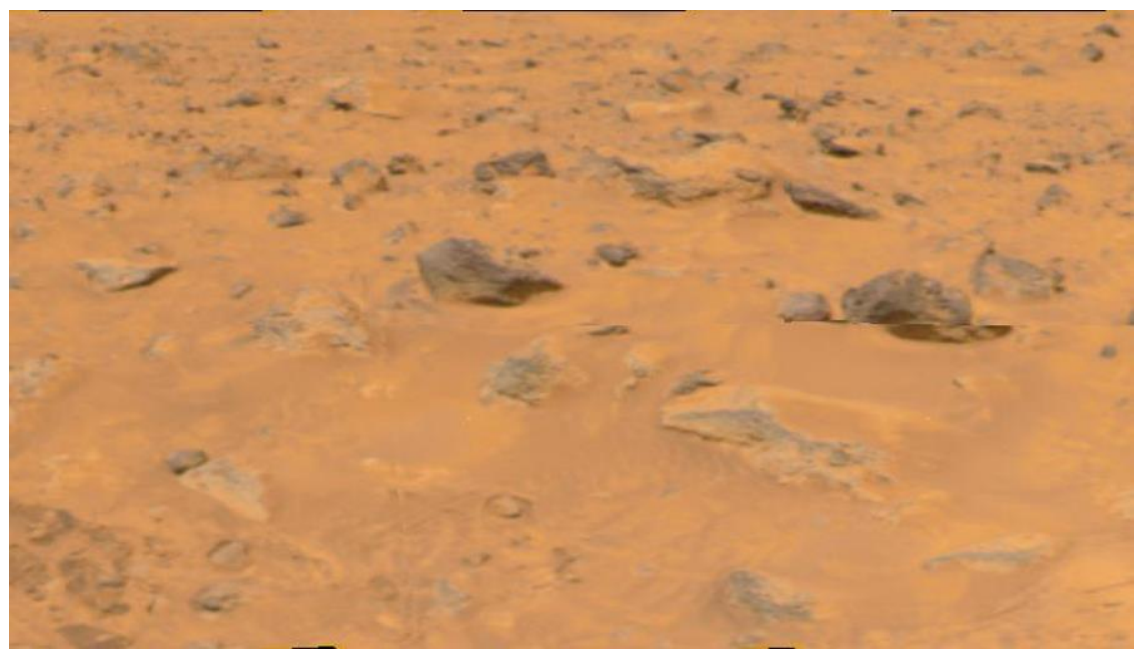

Figure 1: Mars terrain (Wild, 2017) 


\section{How to Change Mars to meet Human Needs}

What do humans need to survive? We have many things on Earth to support our human lives, and many of them must be provided on Mars for human beings to survive. The first need is that we need oxygen. Oxygen is crucial because humans will die in minutes without it. 21 percent of Earth's atmosphere is made of oxygen, and humans require at least 19.5 percent oxygen in the air (NASA, Global climate change, 10 interesting things about air, 2016). Another requirement is that we need gravity. Our body, without any gravity for extensive periods will become weak and unhealthy. Bones are especially affected, and they become weak and more fragile without gravity (CSA, 2018). In addition, food is important for human survival. Food must be nutritious and attractive in order for everyone to be fed in a Mars colony. Along with food, adequate water supply is essential for colonists because human bodies require water for metabolism to survive. If colonists are not provided with water, they could live a maximum of 3-4 days. Water is also needed for growing plants and raising animals.

Stress could be a problem for living in space. Stress can be caused by heavy work, enclosed spaces, and isolation. Stress can cause low energy, headaches, and furthermore cause being more vulnerable to disease, or insomnia. Isolation is a big psychological problem as colonists will stay in small groups, especially during the early days of colonization. Isolation is a main inflictor of stress, so colonists must find ways to reduce it. One way is for colonists to contact Earth regularly to lower the effects of isolation.

We need to make many changes on Mars to have humans be able to live there. First, we need to generate enough oxygen inside our habitats on Mars before we change the entire Mars' atmosphere. There are multiple ways to generate oxygen. The most common way is to split water in a process called electrolysis, which is the water being split by electricity into hydrogen and oxygen gases. Another way is using an "oxygen candle" that uses sodium chlorate and iron powder heated to 600 Celsius to create oxygen. Both ways require a large amount of energy in order to overcome the force of chemical bonds between hydrogen and oxygen atoms in water $(\mathrm{H} 2 \mathrm{O})$ molecules. We can create oxygen using plants, but this is very hard on Mars since it needs to make Mars habitable for plants first. Besides, it would take up too much space on Mars and it is a very slow process. There is a more promising way recently discovered by scientists in Japan and published in Nature. They found a photocatalytic water splitting technique that is able to achieve $96 \%$ efficiency to produce oxygen and hydrogen at room temperature (Tsuyoshi Takata, 2020). This photocatalytic process requires sunlight and water, as illustrated in (Figure 2), which are already abundant on Mars. Using the photocatalytic technique, oxygen can be created on Mars in an efficient and economical way. 


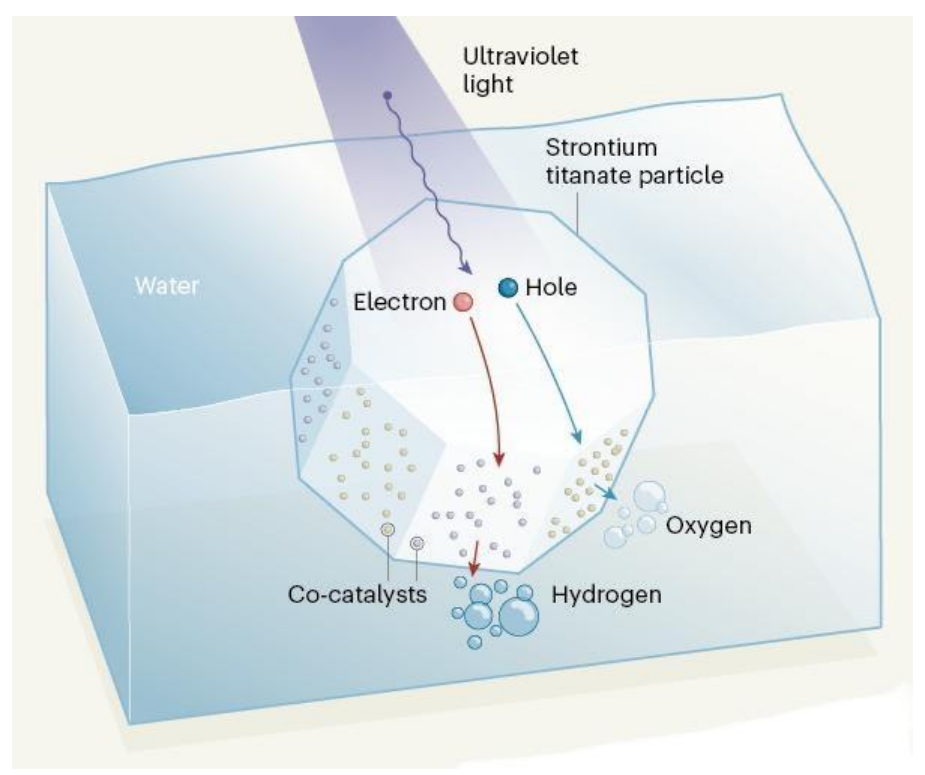

Figure 2: Photocatalytic water splitting (Tsuyoshi Takata, 2020)

The atmosphere temperature on Mars needs to be adjusted in order for humans to live properly on the red planet. Currently, it is too cold for humans to live on Mars without proper protection. Although the habitat can be warmed inside by solar power, it is an average temperature of $-81 \mathrm{~F}$ degrees outside the habitat, while the normal human body temperature needs to stay around 97F. We would have to raise atmosphere temperatures by emitting greenhouse gasses like water vapor, carbon dioxide, and methane, and this process is called "Terraforming" (Figure 3). Terraforming would result in a thicker atmosphere and eventually raise temperatures on Mars. The thicker atmosphere also helps to reduce space debris, like a meteor, from hitting Mars' surface. The Terraforming process will include an artificial magnetic field to block cosmic radiation. Many people say Terraforming is the most ideal solution to colonize Mars, but the process may take centuries to complete. Terraforming will change Mars temperature to a range like Earth's and the plan will include an artificial magnetic field to stop the radiation and debris. If we take fully colonizing Mars as our long term and ultimate goal, we will have to rely on the Terraforming process.

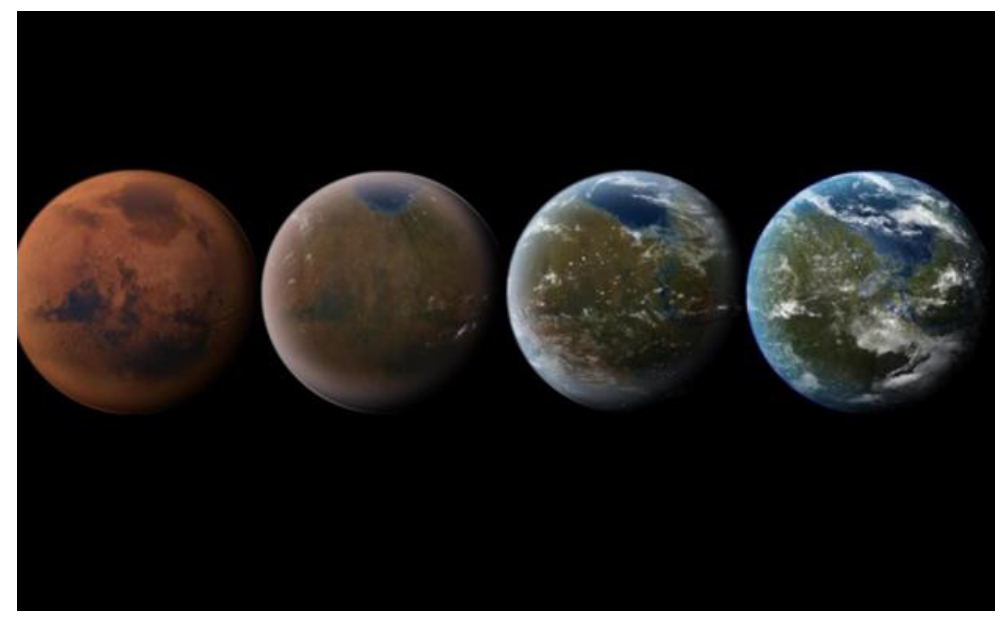

Figure 3: Mars before, during, and after ideal Terraforming (Kenneth Fong, 2017) 
Before Mars is terraformed, colonists probably must live in small groups of habitats and have limited access to the rest of Mars. These habitats will be heated by energy gotten from solar panels or other sources, like the wind. The habits will be centered by the shelters that colonists live in. Though instead of normal houses on Earth, colonists need special shelters that must meet many extra requirements, such as blocking cosmic radiation since Mars is not fully terraformed yet. If the shelters must be transported from Earth, they need to be compact for transport but also be expandable once they arrive at Mars. An idea is to use an inflatable structure such as NASA's Mars ice home, as shown in (Figure 4). It is a structure with sleeping quarters, a greenhouse, airlocks, and ice to block radiation. These structures will not be too big since they need to be pressurized from inside. Similar habitat prototypes (Figure 5), have these features on them.

Another idea is that Mars colonists can dig caves as homes, as humans did in early history on Earth (P. Boston, et. al). The caves would only need very limited materials to be transported from Earth. Therefore, they can be built quickly and economically. The caves naturally can shield cosmic radiation. A more recent idea is to use a 3D printer to "print" homes on Mars using a mixture of Martian soil and water. The prototypes of 3D printed shelters could be in many shapes, even though most designs are low, some are tall like MARSHA, as shown in (Figure 6). MARSHA is a NASA 3D Printed Habitat Challenge winner, it stands upright and tall on the Martian surface with highly human-centric, habitable spaces tuned to the demands of a Mars mission. It can be constructed of biodegradable and recyclable materials, such as basalt composite, a natural mineral found on Mars in great numbers, and is found to be 10 times stronger than concrete. The MARSHA design is already under testing on Earth, and its prototype called "TERA" has been built and tested, as shown in (Figure 7).

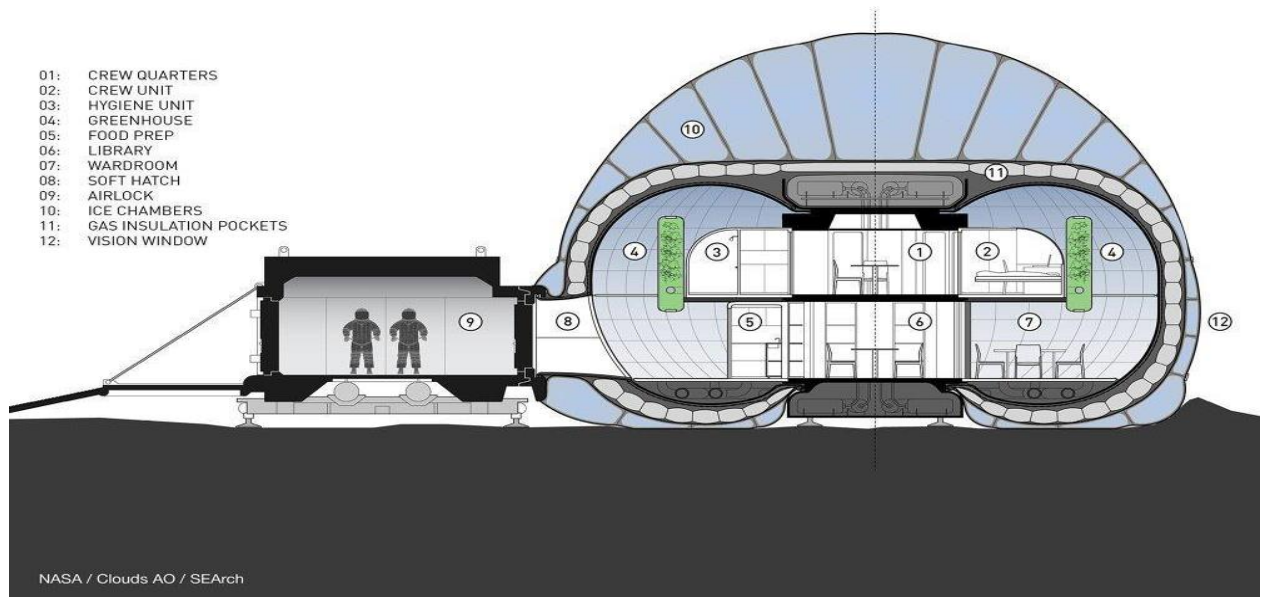

Figure 4: Mars Ice Home 


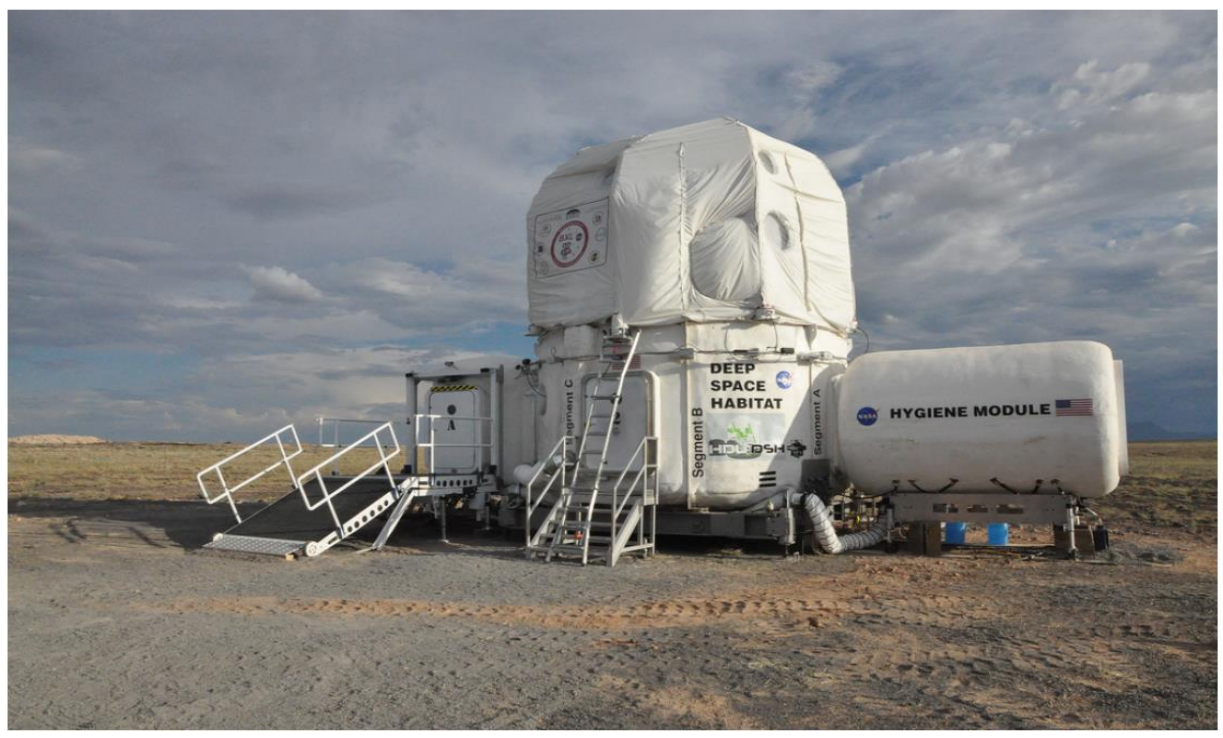

Figure 5: A Prototype of a Habitat Being Tested on Earth (Kenneth Fong, 2017)

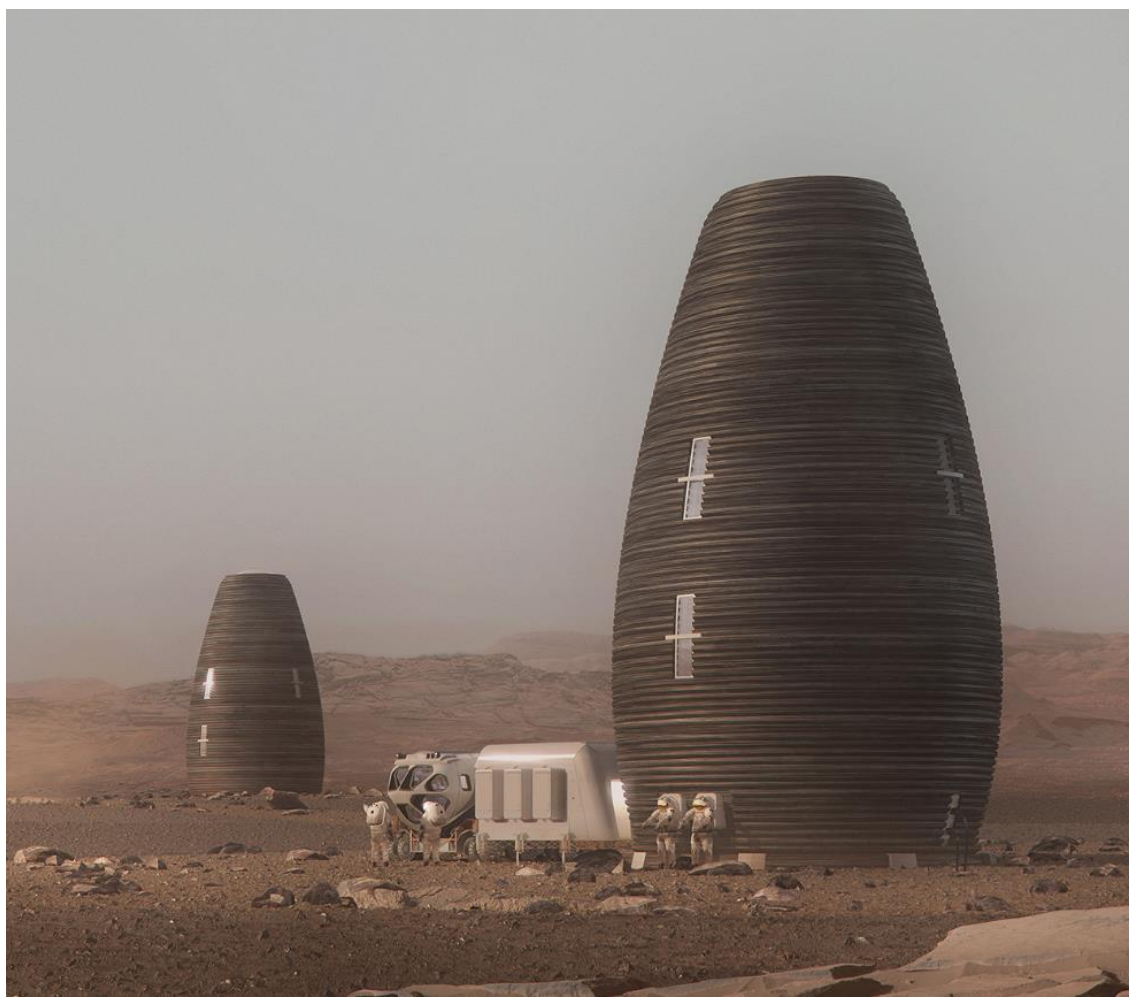

Figure 6: MARSHA 


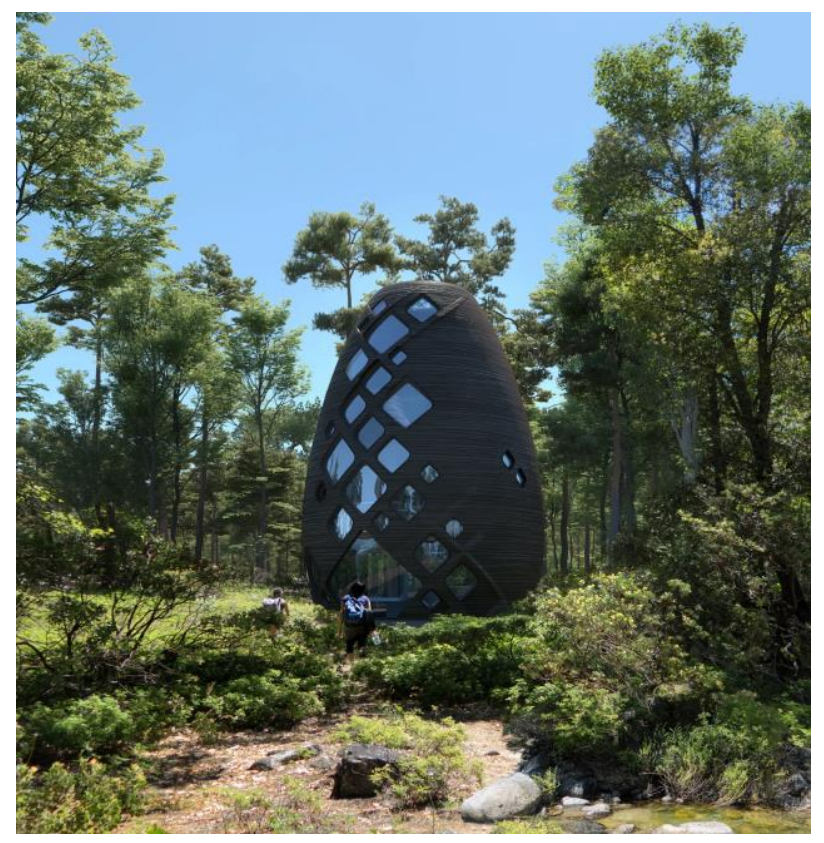

Figure 7: TERA cabin

Everyone knows that water is an essential resource for humans to survive. Luckily, water is not rare on Mars (NASA, 2015). Water could be very abundant on Mars, but we are just not exactly sure where its location is. Having water is one of the most important reasons that we choose Mars instead of any other planet. The ice caps of Mars have a high chance of containing water in its solid form. Water could be underground in areas, like near ice caps. We already know areas that have a good chance of having water, and those almost certainly have water (Figure 8 ). Water could be acquired by digging wells or melting ice caps. These areas with water would be prospect spots for colonies. The water can also support electrolysis or photocatalytic water splitting techniques to generate oxygen. Scientists are working on sending more rovers to confirm water existence in certain areas on Mars.

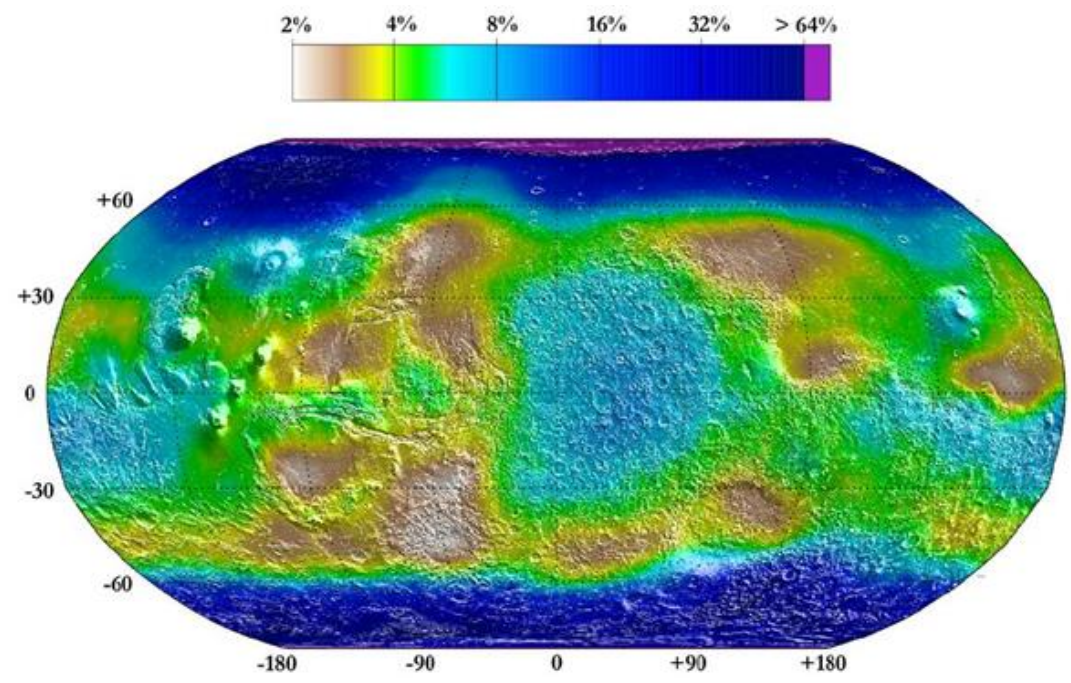

Figure 8: possible areas that have water (Kenneth Fong, 2017) 
Besides the water, colonists need to eat too, but there is no food ready on Mars. Mars colonists should not rely on food supply from Earth. Why? It would be too costly and risky to do that. A regular rocket that launches to a space station on Earth's orbit costs millions of dollars. And the rocket to deliver supplies to Mars would be much more expensive, about 4-6 billion dollars for a very limited amount of food supplies. Moreover, if the rocket fails to deliver food supplies to Mars, the colonists will be in great danger of starvation. Finally, there are only 4-time windows a year to launch a rocket from Earth to Mars. It takes 6 months to reach Mars (SpaceX, 2020). Therefore, colonists will have to find a way to grow their own food on Mars to survive. Foods like potatoes, lettuce, tomatoes, and snap peas can be grown in greenhouses using hydroponic, aquaponic, vertical farming, and aeroponic techniques. Vertical farming is important since there is no space for lots of crop fields. All of these techniques are verified to be feasible and efficient.

Aquaponics would be particularly efficient in this case, because it keeps recycling water and lets both fish and plants thrive, as in (Figure 9). This technique makes the most food with the least materials and waste, since both organisms produce food for each other, like an ecosystem. The other techniques require lots of maintenance, while hydroponics requires little care to grow. Fish are helpful by supporting aquaponics. The crops can produce oxygen as they grow, which supports oxygen production within the habitat. In addition to plants, it is also likely for colonists to raise animals such as quails, goats, rabbits, and fish for colonists to eat. It is believed that specific crops can grow in microgravity, and a recent study was done by NASA proved that to be true (Herridge, 2019). The study, which was called Veg-01, and it was tested on the International Space Station or ISS. The experiment consisted of growing romaine lettuce vertically on rooting "pillows" with red and blue LED lights for photosynthesis. This indicates that plants can be grown on Mars even with microgravity, and the rooting pillows are a possible way to grow crops vertically. Furthermore, the study shows that red and blue led lights can be used to grow plants, which means that no sunlight is needed to grow the plants on Mars. That is a beneficial factor of growing plants because sunlight is weaker on Mars. Psychological benefits were found in growing the romaine lettuce, along with the health benefits of eating the lettuce. Having a piece of the Earth while living on Mars was found to reduce isolation and stress by a considerable amount.

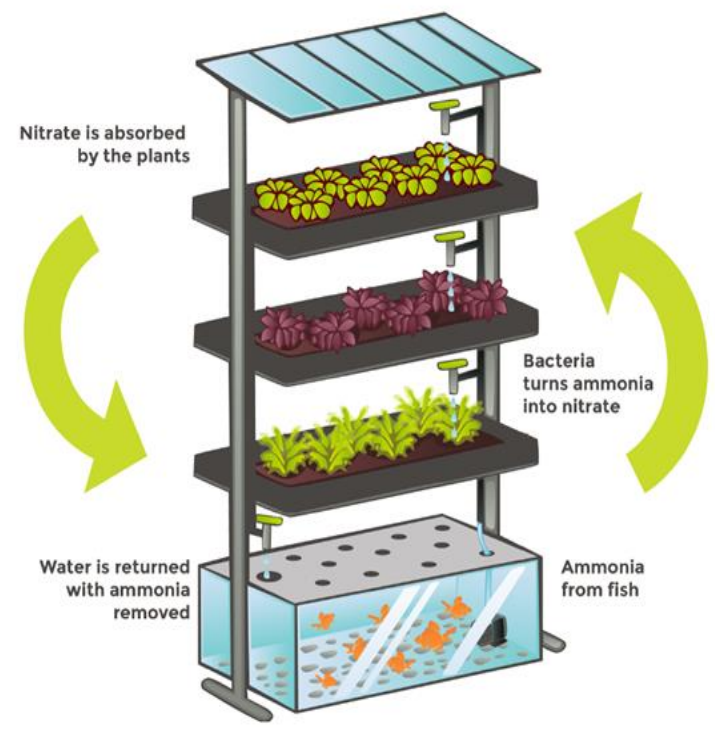

Figure 9: the aquaponics system (Kenneth Fong, 2017) 
Energy is another important to keep habitats functional. Energy could be acquired by using solar panels, wind farms, or nuclear reactors. Solar panels are not a very efficient way of generating energy because Mars only gets $589 \mathrm{~W} / \mathrm{m} 2$, while Earth gets $1367 \mathrm{~W} / \mathrm{m} 2$. The average wind speed on Mars is about 7 meters per second (Mersmann, 2015). Compared to Mars, Earth's wind speed is only 4 meters per second average. This means wind farms can be more effective on Mars and produce more energy. Especially during dust storms, wind speeds on Mars can reach 17-30 meters per second. Wind farms can be a replacement for solar panels when dust storms occur because dust can block the light that the panels require to produce energy.

Another way to produce energy is nuclear energy. It can be used when there is already a big colony on Mars since it is very efficient, but it's hard to assemble. Uranium already exists on Mars, so it will not need to be transported. Nuclear power will not be affected by dust storms and it doesn't require much space, making it very reliable. All these energy-generating processes require certain maintenance, for example, sand from a dust storm could jam a turbine in wind farms. Energy loss could result in blackouts for the colony, so proper maintenance is required if we use solar panels, wind farms, or nuclear power plants to create energy.

Communication is a major problem while going to Mars and during the colonist's stay. (NASA, Moving around Mars) It takes about 20 minutes for a message to get from Earth to Mars. Messages carrying urgent information would be delayed. As a result, phone calls to Mars wouldn't be practical. Colonists would have to Text, Email, or "WhatsApp" to communicate while on Mars. But during a 2-month period, people on Earth cannot communicate with Mars because the sun blocks the signals from reaching Mars (Wall, 2015) (Figure 10). This event happens every 2 years. So far, we have no solution to this problem.

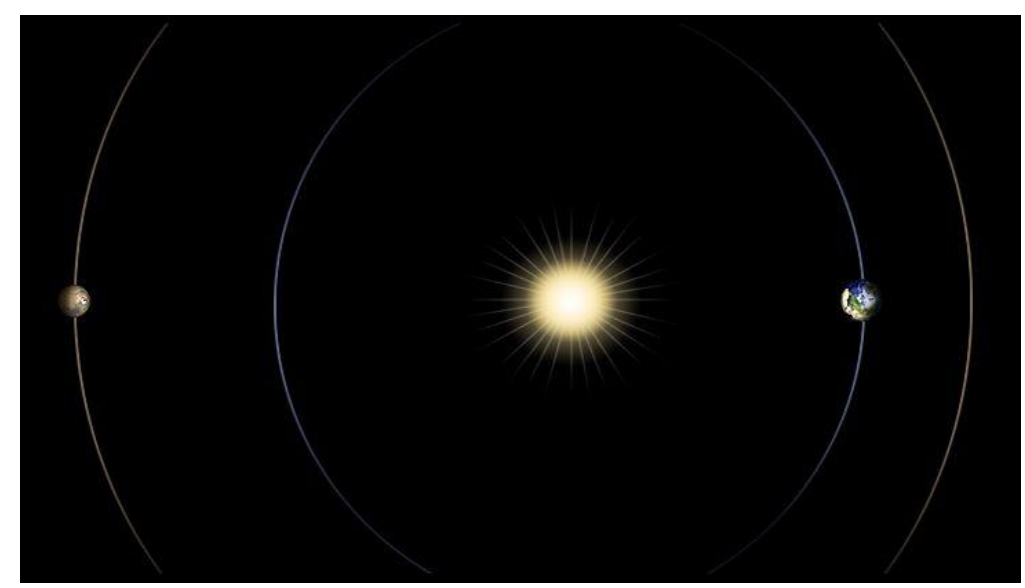

Figure 10: The sun blocks communication between Mars and Earth (Wall, 2015)

\section{Conclusion}

Because of the many solutions and technological advancements humans made recently, it is very possible to meet NASA's goal of putting a man on Mars by 2030. This is so because basic human needs like air could be created in feasible ways. All the colonist's needs can be met with today's technology so we can colonize Mars soon, even though some problems still exist like communication blackouts. 2030 is also a good time to launch a rocket to Mars because Mars is the closest then in 100 years. This means less distance is to be covered, and less fuel is required for the trip to Mars. Exploration using humans on Mars will be a 
major accomplishment. Mars could be a backup home in case Earth is destroyed, or it can provide a pit stop for further space exploration. It could deepen knowledge and understanding of other planets we could possibly colonize, like Titan. Mars is also important to colonize because it can help develop new technology in the process of getting there. We will advance in new technologies like air production, vertical farming, and many other techniques used to colonize Mars. Advances in these fields' technology could soon change things on Earth too, because many things that we use in everyday life came from ideas used in space exploration, like memory foam. Colonizing Mars could cost a lot, but we should still work towards it because it is very rewarding to do so.

\section{References:}

CSA. (2018). What happens to bones in space. From Canadian Space Agency: https://www.asccsa.gc.ca/eng/sciences/osm/bones.asp

Herridge, L. (2019). Meals ready to eat: Expedition 64 crew members sample leafy greens. NASA. From https://www.nasa.gov/mission_pages/station/research/news/meals_ready_to_eat

Kenneth Fong, A. K. (2017). Humanity and Space design: An implementation of a theoretical Martian outpost. WPI. From https://web.wpi.edu/Pubs/E-project/Available/E-project-031517231600/unrestricted/humanity-space-iqp.pdf

Mersmann, K. (2015). The fact and fiction of Martian dust storms. NASA Mars exploration program. From https://Mars.nasa.gov/news/1854/the-fact-and-fiction-of-martian-dust-storms/

NASA. (1997). Martian Terrain. NASA. doi:https://www.jpl.nasa.gov/spaceimages/details.php?id=PIA00653

NASA. (2016). Global climate change, 10 interesting things about air. From NASA: https://climate.nasa.gov/news/2491/10-interesting-things-about-air/

NASA. (2017). NASA confirms that there is water on Mars. From https://www.nasa.gov/press-release/nasaconfirms-evidence-that-liquid-water-flows-on-today-s-Mars/

NASA. (n.d.). Moving around Mars. NASA Mars exploration rovers. From https://Mars.nasa.gov/mer/mission/timeline/surfaceops/navigation/

P. Boston, G. F.-W. (n.d.). Human utilization of subsurface extraterrestrial environments: final report. From http://www.niac.usra.edu/files/studies/final_report/710Boston.pdf

SpaceX. (2020). Missions: Mars. From SpaceX: https://www.spacex.com/human-spaceflight/Mars/

Tsuyoshi Takata, J. J. (2020). Photocatalytic water splitting with a quantum efficiency of almost unity. Nature. doi:https://doi.org/10.1038/s41586-020-2278-9

Wall, M. (2015). Mars Spacecraft Enter Communications Blackout Sunday. SPACE.COM. From https://www.space.com/29576-nasa-Mars-spacecraft-radio-blackout.html

Wild, F. (2017). What is Mars? NASA. DOI: https://www.nasa.gov/audience/forstudents/5-8/features/nasaknows/what-is-Mars-58.html 